\title{
A RADIOTERAPIA DO CÂNCER DE PRÓSTATA: UMA REVISÃO DA LITERATURA DOS PRINCIPAIS AVANÇOS E MÉTODOS DE TRATAMENTO ${ }^{1}$
}

\author{
A PROSTATE CANCER RADIOTHERAPY: A REVIEW OF THE \\ LITERATURE OF MAIN ADVANCES AND TREATMENT METHODS
}

\begin{abstract}
Fagner Brum, Matheus Rizzatti Feron², Carolina Marroni Pulga², Jessica Garcia Dimperio², Andressa Dalmolin ${ }^{2}$, Bruna Garcia Pedrolo ${ }^{3}$, Evamberto Garcia de Góes ${ }^{4}$ e Éder Maiquel Simão ${ }^{5}$
\end{abstract}

\section{RESUMO}

O câncer surge quando uma célula sofre uma instabilidade genética, causando mutações no seu DNA, e passam a se proliferar de forma descontrolada e desordenada, podendo invadir tecidos e órgãos distantes, gerando as metástases. O câncer de próstata é a neoplasia mais comum em homens acima de 40 anos, tendo entre os principais fatores de risco idade avançada, histórico familiar, fatores ambientais, sedentarismo e alimentação rica em gorduras e pobre em fibras e verduras. Uma das principais formas de tratamento, a radioterapia, usa radiação ionizante de forma controlada, procurando uma entrega de dose direcionada às células doentes, afetando o mínimo possível as células sadias em torno do tumor. O objetivo desta revisão foi avaliar a associação entre radioterapia e câncer de próstata, bem como os avanços usando a radioterapia. Os dados para esta revisão foram obtidos através de buscas nas bases de dados Pubmed de artigos publicados nos últimos 10 anos usando os descritores câncer, próstata, tratamentos, radioterapia, novas terapias de radioterapia, evolução em tratamentos de próstata. Houve um grande avanço tecnológico nas terapias com radioterapia, onde a principal delas é a IMRT, pois sua modulação de dose protege de forma mais eficiente os órgãos de risco, como bexiga, reto e pelve. Estudos com códigos de Monte Carlo também apontam o uso de nanopartículas de ouro radiotivas como tratamento promissor para o câncer de próstata.

Palavras-chave: neoplasia, novas terapias, radiação ionizante.

\section{ABSTRACT}

Cancer arises when a cell undergoes genetic instability, causing mutations in its DNA, and they proliferate in an uncontrolled and disordered way, being able to invade distant tissues and organs, generating the metastases. Prostate cancer is the most common neoplasm in men over 40 years of age, with the main risk factors being advanced age, family history, environmental factors, sedentary lifestyle and high fat and low fiber and vegetable intake. One of the main forms of treatment, radiotherapy, uses ionizing radiation in a controlled manner, looking for a dose delivery directed at diseased cells, affecting as little as possible the healthy cells around the tumor. The aim of this review was to evaluate the association between radiotherapy and prostate cancer, as well as the advances using radiotherapy. The data for this review were obtained through searches in Pubmed databases of articles published in the last 10 years using the descriptors cancer, prostate, treatments, radiotherapy, new radiotherapy therapies, evolution in prostate treatments. There has been a great technological advance in radiotherapy therapies, where the main one is the IMRT, since its dose modulation more efficiently protects the ${ }^{1}$ Trabalho desenvolvido na disciplina de Radioterapia.

${ }^{2}$ Acadêmicos do Curso de Física Médica - Universidade Franciscana - UFN. E-mail: kk_mp@hotmail.com

${ }^{6}$ Acadêmica do Programa de Pós-Graduação em Nanociências - Universidade Franciscana - UFN. E-mail: brunapedrolo@ gmail.com

${ }^{7}$ Docente do Curso de Física - Universidade Federal do Rio Grande - FURG. E-mail: evambertogoes@gmail.com

${ }^{8}$ Orientador - Universidade Franciscana - UFN. E-mail: edersimao@gmail.com 
organs of risk, such as bladder, rectum and pelvis. Monte Carlo code studies also point to the use of radioactive gold nanoparticles as a promising treatment for prostate cancer.

Keywords: neoplasia, new therapies, ionizing radiation.

\section{INTRODUÇÃO}

O câncer é definido como o crescimento desordenado de células, que provoca mutações no DNA. Com a divisão desordenada, as células tendem a ser agressivas e incontroláveis, determinando a formação e crescimento de tumores. Os tumores provocam o crescimento excessivo das células anormais no local, podendo ocorrer metástase, ou seja, espalhamento das células em outras regiões do corpo (SOHAIL et al., 2019).

O câncer de próstata surge em homens com idades superiores a 40 anos. Dentre os homens mais afetados, estão os afro-americanos e os homens que possuem casos desse tipo de câncer na família. Ela é a neoplasia mais comum no Brasil, compondo 70 novos casos para cada 100 mil homens. Na região sul esse tipo de doença aparece em 19,3\% dos casos de câncer ( COSTA et al., 2015).

A radioterapia é um tipo de tratamento utilizado para destruir as células do câncer. Este tratamento utiliza feixes de radiação de alta intensidade produzidos por radioisótopos (braquiterapia) ou acelerador linear (teleterapia). O tratamento radioterápico pode ser associado a outros tipos de tratamento como a quimioterapia e a cirurgia. A cirurgia é indicada para a remoção do volume que engloba o câncer. A quimioterapia utiliza medicamentos administrados ao paciente via intravenosa, oral, intramuscular, entre outros, para que estes se misturem a corrente sanguínea do paciente e auxiliam na destruição das células cancerosas, para que as mesmas não se espalhem. Além destes tratamentos, a utilização de novas tecnologias permite ainda o uso de tratamentos personalizados, tais como a imunoterapia, e terapias usando direcionamento de fármacos, como é o caso de fármacos nanoencapsulados (FARIA et al., 2001).

A radioterapia visa a cura ou proporciona uma melhor sobrevida ao paciente, quando não há condições de cura. Com altas doses, a radiação ionizante danifica o DNA das células, podendo provocar a morte ou reduzir o crescimento de células cancerígenas (KHAN; GIBBONS, 2014).

Com a evolução dos equipamentos usados em radioterapia foi possível o desenvolvimento de novas técnicas de tratamento que possibilitaram uma melhor distribuição de doses de radiação no volume de tratamento e preservação dos órgãos sadios adjacentes. A radioterapia conformacional tridimensional (3D-CRT) foi desenvolvida como uma técnica de planejamento para suprir essa necessidade, ou seja, uma melhor utilização da radiação durante o tratamento. Para o tratamento de câncer de próstata há uma nova metodologia de planejamento emergente, que demonstrou uma melhora na 
toxicidade dos órgãos de risco, como também, uma entrega de doses mais elevadas proporcionando dessa forma um efeito curativo maior na região da próstata, essa técnica é conhecida como radioterapia de intensidade modulada (IMRT) (ONCOGUIA IMRT, 2014).

O presente estudo tem como objetivo desenvolver uma revisão sistemática da literatura, voltada às análises evolutivas dos avanços usandos em radioterapia indicada para o tratamento do câncer de próstata. O material revisado foi estudado a partir da técnica de análise de conteúdo temática. E os resultados estão apresentados na forma de capítulos.

\section{METODOLOGIA}

\section{Fonte de dados e pesquisa}

Os dados para esta revisão foram pesquisados no PubMed. Todos os estudos sobre a associação entre câncer de próstata e evolução nos tratamentos em radioterapia incluíram os termos de pesquisa em inglês: "cancer", "prostate”, "treatments", "radiotherapy”, "new radiotherapy techniques" e "evolution in prostate treatments" como descritores.

\section{Seleção dos estudos}

Através da pesquisa por "cancer" foram encontrados 3.887.108 artigos. Ao adicionar o termo "prostate", esse número foi reduzido à 149.674 artigos. Quando adicionado o descritor "treatment" foram encontrados 90.939 artigos. Ao adicionar o termo "radiotherapy", esse número foi reduzido à 19.882. Quando adicionado o termo "radiotherapy and new radiotherapy techniques" foram encontrados 2.456 artigos. Ao adicionar o termo "evolution in prostate treatments", esse número foi reduzido à 26 artigos. Desses 26 artigos, por fim, foram selecionadas as publicações entre o período de 2010 a 2019, restando 16 artigos. Foram examinados os títulos, e então selecionados 10 artigos, para leitura dos respectivos resumos. Desses, apenas 1 artigo foi utilizado, pois abordava o tema selecionado para a construção desse trabalho. A seleção do estudo pode ser melhor visualizada no fluxograma da Figura 1.

Figura 1 - Fluxograma da seleção do estudo. 


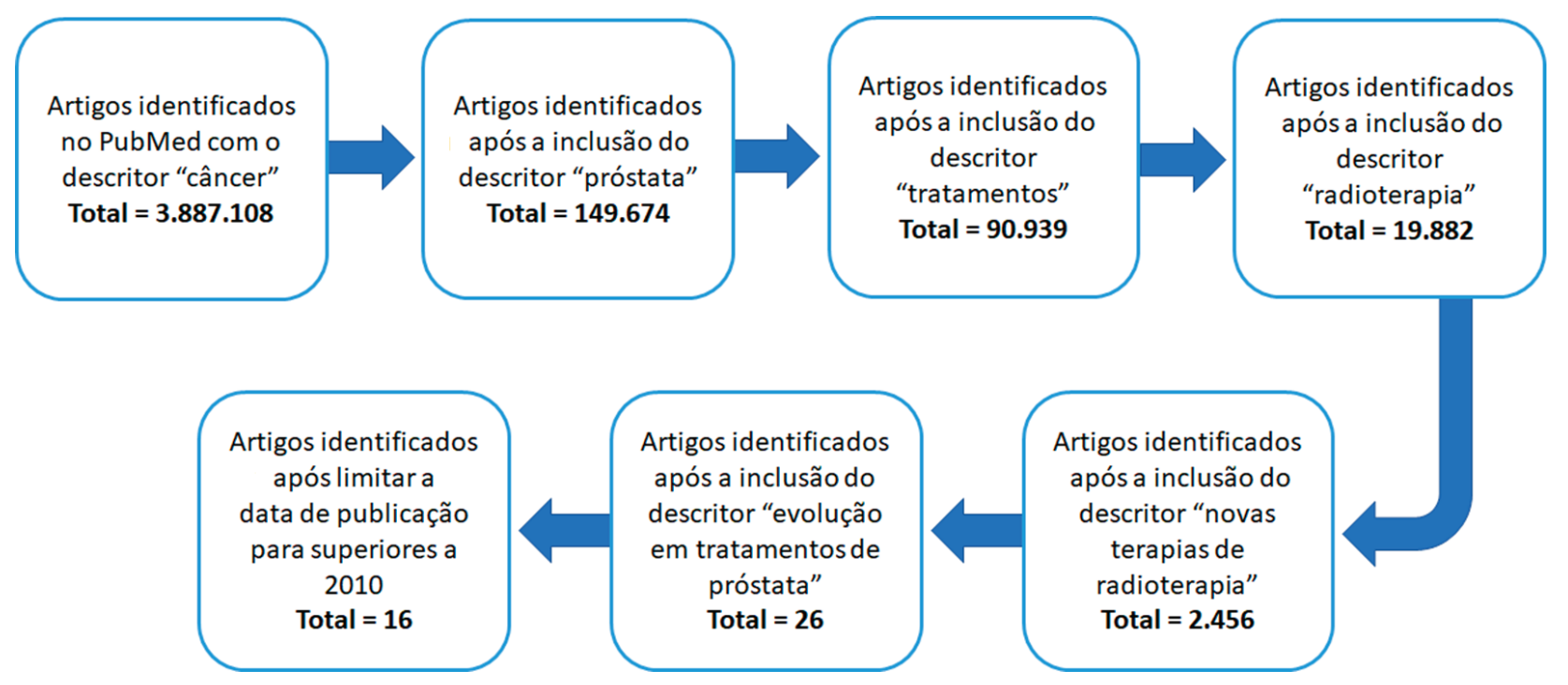

Fonte: Construção dos Autores.

\section{O Câncer de Próstata}

As células cancerígenas, são células defeituosas que crescem de forma anormal no organismo, formando uma massa tumoral tomando o lugar das células saudáveis em tecidos ou órgãos. Esses tumores interferem no funcionamento saudável do órgão em que estão instalados, podendo se espalhar pela corrente sanguínea e pela cadeia linfática para outros órgãos. Atualmente, tem-se catalogado mais de 200 tipos de câncer diagnosticados, decorrentes de fatores externos e internos como cigarro, idade, álcool, hormônios, infecção crônica e radiação (SOHAIL et al., 2019).

Um dos cânceres mais comum em homens é o câncer de próstata, que atinge em sua grande maioria, homens acima de 40 anos. A próstata é uma glândula masculina que se localiza na parte baixa do abdômen logo abaixo da bexiga e à frente do reto. Os tumores que se desenvolvem na próstata, apresentam algumas características, como a heterogeneidade, isto é, com características morfológicas e fenotípicas distintas. No Brasil, o câncer de próstata é o segundo mais comum entre os homens, ficando atrás somente do câncer de pele. O crescimento do tumor de próstata, na maioria das vezes, é muito lento, levando a um diagnóstico tardio. $\mathrm{O}$ aumento nas taxas de diagnósticos desse tipo de câncer pode ser justificado pela evolução dos métodos diagnósticos, pela melhoria na qualidade dos sistemas de informação dos países e pelo aumento na expectativa de vida. Existem dois tipos de diagnósticos para o câncer de próstata: exame de toque e exame de PSA (prostate-specific antigens ou antígeno específico da próstata), que é um exame de sangue usado para medir a quantidade de uma proteína produzida pela próstata (COLLINS et al., 2005).

Existem fatores de riscos para desenvolver o câncer de próstata, podendo ser a idade, o sobrepeso ou o histórico de câncer de próstata na família. Para evitar, ou minimizar os riscos de desenvolver esse tipo de câncer, é necessário que o homem tenha hábitos de alimentação saudáveis, pratique exercí- 
cios e faça exames periodicamente para a monitoração da próstata (MINISTÉRIO DA SAÚDE, 2019).

\section{Avanços em Radioterapia}

A Radioterapia é um tratamento curativo ou paliativo para cânceres utilizando radiação ionizante de alta intensidade (com feixes de fótons e elétrons que possuem energias superiores a 8 $\mathrm{MeV})$. O número de aplicações necessárias varia de acordo com as características específicas de cada paciente e tumor e do local em que ele se encontra. Pode ser feita juntamente com a quimioterapia ou cirurgias. A radioterapia é subdividida entre teleterapia e braquiterapia, em que na primeira o tratamento é feito utilizando radiação produzida em aceleradores lineares ou telecobaltoterapia e na segunda utilizando fontes de radiação seladas (KHAN; GIBBONS, 2014).

Para que o volume tumoral (alvo) seja completamente eliminado várias aplicações são necessárias e em vários ângulos de incidência, assim o tecido sadio circunvizinho será preservado ao máximo e as células cancerosas receberão a maior dose possível. Durante a radioterapia o tumor é atingido por feixes de radiação ionizantes, que ao interagirem com a matéria causam efeitos biológicos como eritemas, efeitos genéticos, catarata, câncer e até mesmo a morte, dependendo da dose recebida ou acumulada. A ionização pode provocar quebras na estrutura molecular causando danos irreversíveis ou não as células, estes danos podem inviabilizar a divisão celular ou até levar à morte celular (OKUNO; YOSHIMURA, 2010).

Com o passar do tempo a radioterapia obteve um incremento em suas formas de tratamento e técnicas utilizadas desde os tipos de equipamentos a métodos de planejamentos. Começando com os equipamentos de Cobalto 60, e a utilização do planejamento conhecido atualmente como radioterapia convencional baseado apenas em duas dimensões (2D), onde o volume de tratamento é delineado sobre imagens de radiologia convencional (radiografias que possuem apenas as dimensões de um plano). Com o avanço tecnológico o planejamento 2D perdeu espaço para planejamentos mais avançados que garantem uma maior exatidão e conformação com os volumes tumorais. Porém o planejamento 2D ainda é utilizado em técnicas que envolvam tratamentos paliativos, ou tratamentos com doses menores quando comparadas a novas técnicas de planejamento existentes (KHAN; GIBBONS, 2014).

Com o advento dos aceleradores lineares houve uma transição entre a tecnologia dos equipamentos emissores de radiação através do radioisótopo cobalto (Energia de 1,22 MeV) para esses novos equipamentos com energias superiores a $8 \mathrm{MeV}$. Eles permitem o uso de uma maior dose de radiação e a não utilização de uma fonte radioativa, dessa forma aprimorando processos de proteção radiológica como os demais processos logísticos envolvidos com essas máquinas. Os equipamentos de telecobaltoterapia utilizam radiação gama originada da fonte radioativa presente no interior do equipamento (exemplo Cobalto 60 ou telecobaltoterapia), que no momento do tratamento é exposta através de um mecanismo físico da máquina, nos aceleradores lineares há dois tipos de radiação uti- 
lizadas, os feixes de raios $\mathrm{X}$ de alta energia e os feixes de elétrons (ambos com energias superiores a $8 \mathrm{MeV}$ ). Os feixes de raios $\mathrm{X}$ por serem ondas eletromagnéticas atingem maiores profundidades de tratamento, enquanto que nos feixes de elétrons são partículas que possuem massa e por esse motivo possuem uma menor penetrabilidade, com isso são basicamente utilizados para tumores mais superficiais (CONNELL, 2009).

A radioterapia quando utilizada em conjunto com o planejamento tridimensional conformacional ou simplesmente 3D revolucionou o tratamento do câncer, já que nessa modalidade de planejamento pode-se aumentar a dose no volume tumoral ao mesmo tempo que permite uma redução de dose de radiação nos tecidos sadios adjacentes, dessa forma diminuindo de forma considerável os efeitos colaterais. Isso só foi possível com a evolução da radiologia como um todo, a popularização da tomografia computadorizada e até mesmo em alguns casos a ressonância magnética que proporcionou que as imagens da região tumoral sejam adquiridas em três dimensões, tendo uma noção volumétrica do tumor com uma maior precisão e também dos órgãos de risco ao redor da lesão. Principalmente a tomografia contribui para esse processo, pois através dos números de CT (número da densidade de Tomografia Computadorizada), isto é, os valores da escala de Hounsfield associados com as diferentes densidades encontradas no meio biológico permitiram que os algoritmos dos programas de planejamento computadorizados possam realizar os cálculos estimando os valores de radiação que cada órgão e volume tumoral irão receber durante o tratamento, processo esse que era inviável com a telecobaltoterapia e o planejamento 2D, onde era baseado em imagens de raios X (ONCOGUIA IMRT, 2014).

A radioterapia de intensidade modulada (IMRT) é mais uma forma de teleterapia que como a 3D permite uma entrega de dose com uma alta precisão no volume alvo de tratamento, ao passo que diminui os efeitos colaterais, tendo em vista que protege com maior eficiência órgãos de riscos ou tecidos sadios próximos ao tumor. Em comparação ao planejamento tridimensional conformacional a IMRT permitiu uma evolução no sentido de aumentar doses no tumor e diminuir nos órgãos de risco, embora em muitos casos essas diferenças acabam sendo pequenas, em determinados tumores e lesões. Há uma diferença considerável na capacidade do sistema de planejamento de conformar com maior precisão sobre o volume tumoral, buscando sempre a máxima redução da toxicidade envolvida no tratamento utilizando a radiação ionizante. Em tratamentos onde as lesões ou tumores estão próximos a estruturas ou órgãos nobres, normalmente indica-se a utilização dessa técnica de planejamento, pois pode-se obter uma conformação com maior precisão. Alguns casos onde o tumor está localizado por exemplo na região pélvica, existe a possibilidade de reduzir o risco de efeitos colaterais de longo prazo no intestino, como também em tumores de cabeça e pescoço, onde pode-se proteger as glândulas salivares e mesmo a medula com uma maior eficácia utilizando-se a IMRT (EINSTEIN IMRT, 2019).

As principais diferenças entre as duas técnicas de planejamento 3D e IMRT são que na primeira após o delineamento do volume de tratamento e órgãos de risco, e a prescrição de dose pelo radioterapeuta o planejamento 3D vai possibilitar feixes de radiação simples conformados com o volume 
tumoral de forma que haja margens nesses campos com o objetivo de abranger as variações que possam ocorrer na configuração da máquina, tal qual no posicionamento do paciente durante o tratamento. Na IMRT o que ocorre é a necessidade de planejar estruturas, isto é, determinar para cada estrutura o valor de dose que respectivamente irão receber, tanto para o volume de tratamento quanto para os órgãos que necessitam ser preservados. O sistema de planejamento define um padrão para aquele paciente em questão e dessa forma o feixe é modulado dependendo do ângulo que estiver incidindo no corpo do paciente, utilizando a tecnologia das múltiplas lâminas que fazem a conformação espacial das estruturas. Em outras palavras o sistema após receber a informação de quais doses cada estrutura irá receber, planeja através de vários algoritmos a melhor conformação possível para que ocorra a maior entrega de dose de radiação no tumor protegendo os demais órgãos. O físico médico faz a verificação dosimétrica de cada planejamento, garantindo assim que a dose planejada seja entregue corretamente aos pacientes, essa ocorre através da dosimetria que o físico realiza de cada planejamento.

A técnica de IMRT é indicada principalmente quando o planejamento 3D-CRT, não consegue proteger de forma eficiente os órgãos de risco, isto é, quando às restrições de doses não são alcançadas. Os dois principais tratamentos que normalmente respondem melhor com a IMRT são os de crânio, pois há uma melhor proteção às vias ópticas e o tronco cerebral, e os tratamentos de próstata, devido a melhor proteção do reto e bexiga, principais órgãos de risco no câncer de próstata (OLIVEIRA, 2014).

Apesar de todos os avanços no diagnóstico tridimensional, a intensidade modulada da radiação externa, as toxicidades remanescentes da radioterapia no reto e na bexiga são uma grande preocupação. Assim, é importante desenvolver novas abordagens para aumentar a eficácia da radiação no câncer de próstata. Sendo assim, o grupo de Abbasi (2019) publicou um estudo relatando o uso do código MCNP4C Monte Carlo para investigação do efeito do radiofármaco Samário-153 (Sm-153) composto por nanopartículas de ouro 197 (Au-197) no tratamento do câncer de próstata. Além disso, fizeram uma comparação entre os efeitos das nanopartículas de Au-197 nas contribuições de doses simuladas pela próstata. As nanopartículas de Au-197 e as fontes de Sm-153 são uma solução mista que contém 20 mCi de Sm-153 e 10 ml de nanopartículas de Au-197. O Sm-153 é um radioisótopo sintético emissor beta e gama com meia-vida de 46,7 h. O efeito terapêutico do Samário surge da emissão de radioisótopos com meia-vida curta e emissão de partículas desejáveis. Ele emite três partículas beta comuns, incluindo uma energia máxima $\left(\mathrm{E}_{\max }\right)$ igual a $704 \mathrm{keV}(49,4 \%), \mathrm{E}_{\max }$ igual a $634 \mathrm{keV}(31,3 \%)$ e $\mathrm{E}_{\max }$ igual a $807 \mathrm{keV}(18,4 \%)$ que são adequadas para degradar células malignas. Ele também emite dois fótons gama em $\mathrm{E} \gamma=103 \mathrm{keV}(29,2 \%)$ e $\mathrm{E} \gamma=70 \mathrm{keV}(4,7 \%)$ de energia. Portanto, esse fóton liberador de baixa energia permite que os médicos estejam cientes da distribuição e quantidade do radionuclídeo. Além disso, a curta meia-vida do Sm-153 torna seu radionuclídeo adequado para uma rápida liberação do corpo (ABBASI; SADIKOGLU; HASSANZADEH, 2019).

A interação do radionuclídeo Sm-153 com o tecido da próstata ocorre por interação direta de 
fótons gama e partículas beta. A taxa de dose absorvida foi simulada no modelo da próstata $(4 \mathrm{~cm} \times$ $3 \mathrm{~cm} \times 2 \mathrm{~cm}$ ), com valor de $20 \mathrm{mCi}$ do radiofármaco $\mathrm{Sm}-153$ que foi distribuido uniformemente no tecido da próstata. A taxa total de dose absorvida no volume total da próstata e no exterior da próstata é de $76.176 \pm 0,032 \mu \mathrm{Gy} / \mathrm{s}$ e de $22,081 \pm 0,028 \mu \mathrm{Gy} / \mathrm{s}$, respectivamente, onde pode-se observar que a taxa de dose absorvida em todo o volume da próstata é maior do que no exterior da próstata. Isto, é devido ao fenômeno bremsstrahlung de partículas $\beta$ com átomos de Au-197 (ABBASI; SADIKOGLU; HASSANZADEH, 2019). Ainda segundo os autores, o tratamento de nanopartículas de Au-197 para um modelo de amostra de próstata foi realizado com sucesso usando o código Monte Carlo MCNP4C. Nestes cálculos, verificaram que as fontes de Au-197 e Sm-153 podem otimizar a taxa de dose absorvida no tratamento de tumores da próstata. Os resultados desta investigação mostraram que o Au-197 tem papel efetivo na terapia do câncer do ponto de vista teórico. O radiofármaco misto Au-197 e Sm-153 indicou que a taxa de dose absorvida no volume total da próstata a 3\% foi aumentada e o valor indesejável da dose no exterior da próstata a 7\% diminuiu (ABBASI; SADIKOGLU; HASSANZADEH, 2019).

O grupo de Al-Yasiri (2019) também publicou um estudo relatando o uso de nanopartículas de $\mathrm{Au}$ em tratamento de câncer de próstata. O objetivo desta pesquisa foi estimar a distribuição da dose entregue por nanopartículas de ouro radioativas (198AuNPs ou 199AuNPs) ao tumor dentro da próstata humana, bem como aos tecidos normais ao redor do tumor usando o código Monte-Carlo N-Particle (MCNP-6.1.1). Nanopartículas de ouro radioativas estão surgindo como agentes promissores para a terapia do câncer e estão sendo investigadas para tratar o câncer de próstata em animais. Para utilizá-los como uma nova modalidade terapêutica para o tratamento do câncer de próstata humano, são necessárias simulações precisas de dosimetria de radiação para estimar a deposição de energia no tumor e tecidos adjacentes e estabelecer o curso da terapia para o paciente. Utilizou-se um modelo de próstata humana e calculou-se a dose depositada por 198AuNPs ou 199AuNPs no tumor prostático bem como nos tecidos normais que rodeiam a próstata utilizando o código MCNP. Os fantons de água A-150 TEP foram usados para simular os tecidos moles e tumorais. Os resultados mostraram que a dose devido a 198AuNPs ou 199AuNPs, que são distribuídos homogeneamente no tumor, tinham um valor máximo na região do tumor e depois diminuía rapidamente em direção à interface próstata-tumor e órgãos adjacentes. No entanto, a dose depositada por 198Au é significativamente maior do que a dose depositada por 199Au na região do tumor, bem como tecidos normais. De acordo com os resultados do MCNP, as 198AuNPs são uma modalidade promissora para tratar o câncer de próstata e outros cânceres, e as 199AuNPs podem ser usados para fins de imagem (AL-YASIRI; WHITE; KATTI; LOYALKA, 2019).

Em fevereiro deste ano, o grupo de Mountris (2019) publicou um estudo onde o objetivo foi propor uma abordagem de planejamento inverso que possa melhorar a qualidade do planejamento, combinando informações de dose-volume e precisão, sem comprometer os tempos gerais de execução. O planejamento inverso é parte integrante da braquiterapia moderna com baixa taxa de dose. Os sistemas atuais de planejamento clínico não exploram as informações sobre a dose total e usam 
amplamente o formalismo de dosimetria da Associação Americana de Físicos em Medicina, TG-43, para garantir o planejamento clinicamente aceitáveis. Assim, planos sub-ótimos podem ser derivados como resultado da superestimação da dose relacionada ao TG-43 e da não conformidade com os requisitos de distribuição da dose. O mapa de doses foi gerado pela acumulação de núcleos de doses pré-computados de Monte Carlo $(\mathrm{MC})$ para cada local candidato à implantação da fonte. A carga computacional de MC foi reduzida usando a aceleração da unidade de processamento gráfico, permitindo que cálculos precisos de dosimetria fossem realizados no ambiente intraoperatório. O algoritmo de otimização de recozimento rápido de histograma dose-volume (DVH) proposto foi avaliado usando planos clínicos que foram entregues a 18 pacientes submetidos à braquiterapia prostática de baixa taxa de dose. O método gerou planos em 37,5 $\pm 3,2$ segundos com cobertura semelhante da dose da próstata, melhor homogeneidade da dose da próstata em até 6,1\% e menor dose na uretra em até 4,0\%. A inclusão dos requisitos do hisograma dose volume (DVH) permitiu maior controle sobre o resultado da otimização e a qualidade do plano ideal foi aprimorada ainda mais considerando a heterogeneidade do tecido (MOUNTRIS; VISVIKIS; BERT, 2019).

Krengli e colaboradores (2016) realizaram um estudo com o objetivo de comparar a variação diária detectada por duas modalidades de IGRT de imagem não ionizante (imagem de superfície, 3D e ultra-som trans-abdominal, US-3D) para verificar a configuração do paciente com câncer de próstata e as variações de órgãos internos. A radioterapia guiada por imagem (IGRT) é um pré-requisito essencial para a realização de radioterapia de alta precisão. A modulação por intensidade está se tornando o padrão para radioterapia da próstata. Com esta técnica, margens apertadas devem ser aplicadas ao volume alvo para limitar a dose aos tecidos normais. Portanto, é necessário, após a identificação e correção do erro sistemático, localizar com precisão o alvo em cada fração de radiação, a fim de minimizar o risco de falta do alvo relacionado ao erro aleatório. Por esse motivo, o uso de modalidades de imagem sem radiações ionizantes pode incentivar o uso diário de IGRT. O grupo detectou desvios maiores que $10 \mathrm{~mm}$ em um subconjunto relevante da população do estudo, onde essas diferenças parecem ser não apenas estatisticamente significativas, mas também clinicamente relevantes. As diferenças de erros sistemáticos detectados pelas duas modalidades diferentes de IGRT foram bastante semelhantes nas direções AP e CC, mas significativamente diferentes na direção LL. De fato, uma fonte adicional de incerteza na direção do LL pode ser a identificação precisa da borda lateral da próstata pelos US-3D e US para IGRT diária de câncer de próstata. Esses achados sugerem que as duas técnicas podem ser usadas como métodos complementares de controle de qualidade, além de radiografias semanais / feixe cônico, e podem representar uma modalidade diária de IGRT “de baixo custo" e não invasiva para pacientes com câncer de próstata (KRENGLI et al., 2016).

\section{A radioterapia do câncer de próstata}


O câncer de próstata é o tipo de câncer mais comum entre os homens, dessa forma ao decorrer dos anos foram desenvolvidas formas de tratamentos visando a cura para os pacientes, ou uma melhoria na sua qualidade de vida, também conhecidos como tratamentos paliativos. A radioterapia destaca-se como um dos principais tratamentos para o câncer de próstata, podendo ser combinada ou não com outras formas de tratamento. No caso do câncer de próstata descobriu-se uma melhora na sobrevida dos pacientes com o aumento da dose de radiação, no entanto, esses aumentos são limitados pela dose máxima aceita em órgãos de riscos, tais como: bexiga, reto e ossos da pelve. A dose padrão atual para o câncer de próstata está entre 75 Gy e 80 Gy (DEARNALEY, 2016).

Estudos apontam que existem diferenças de dose inerentes a duas técnicas de radioterapia, a radioterapia conformacional tridimensional (3D- CRT) e a radioterapia de intensidade modulada (IMRT). Um estudo realizado utilizou 15 pacientes com câncer de baixo e intermediário risco, que foram tratados com 3D-CRT com a dose convencional de 72 Gy (RCT, 2014). Para cada um dos pacientes foram elaborados dois planejamentos adicionais, ambos utilizando o IMRT, um com 72 Gy e outro com 80 Gy, para comparação de doses nos órgãos de risco no volume alvo e da homogeneização da dose (IMRT, 2014). A dose crítica para o restante foi de V70 (volume recebendo 70 Gy) em $\leq 20 \%$ de todo o volume. Para a bexiga: V70 em $\leq 25 \%$ de todo o volume. Para as cabeças femorais: V50 em $\leq 5 \%$ de todo o volume e para o bulbo peniano a dose média deve ser $\leq 50$ Gy (NASR, 2016). Através de diversos estudos comparativos entre IMRT e 3D-CRT, percebeu-se que há uma ligeira vantagem do IMRT no tratamento de câncer de próstata em relação a outra técnica, devido a possibilidade de utilização de doses relativamente maiores, com a primeira técnica em torno de 75 Gy e 78 Gy, enquanto com o 3D-CRT, entre 70 Gy e 74 Gy. No estudo citado a utilização do IMRT como forma alternativa de planejamento radioterápico em relação ao 3D-CRT, mostrou-se vantajoso, pois o aumento de dose de radiação, não necessariamente aumentou a dose nos órgãos de risco, tendo em vista que para essa neoplasia utiliza-se atualmente doses elevadas de radiação, já que clinicamente justifica-se pela melhor resposta do tratamento, a utilização da IMRT cada vez mais torna-se a opção preferencial, devido a oferecer menor toxicidade ao paciente no pós tratamento quando comparado a técnica de planejamento 3D-CRT (NASR, 2016).

Uma alternativa para a radioterapia convencional, é a próton terapia. Esta nova modalidade de tratamento utiliza, ao invés feixe de fótons de raios X, feixes de prótons. A próton terapia, com o passar dos anos, vem evoluindo juntamente com os avanços tecnológicos. Para gerar os feixes de prótons são necessárias elevadas energias, acarretando dessa forma na necessidade de uma grande estrutura física, aumentando os custos de operação.

A principal vantagem da próton terapia está relacionada ao pico de Bragg do feixe de prótons, possibilitando doses na entrada da pele menores do que os feixes de raios X preservando estruturas e órgãos sadios, pois o pico da dose será no volume tumoral, caracterizando o pico de Bragg. No entanto 
diferentemente da radioterapia convencional a dose não é depositada de forma homogênea, isto é, na próton terapia ocorre o processo de modulação da dose, através da justaposição de picos em diferentes profundidades, dessa forma homogeneizando a dose no volume tumoral (MAZAL, 2010). A figura 2 abaixo, representa um comparativo entre a próton terapia e a radiologia convencional, e às energias necessárias para produzir o feixe de prótons. Observa-se que ao longo da profundidade a dose de radiação se acomula de forma abrupta. A profundidade de interação máxima do feixe de prótons com o tecido, irá variar de acordo com a energia do feixe. Com isso esse método de tratamento do câncer é utilizado para tratar tumores mais profundos. Inicialmente a próton terapia foi usada para tratar tumores cerebrais e recentemente (maior que 20 anos) ela tem sido usada no tratamento do câncer de próstata, mama e fígado. No tratamento da próstata ela é usada para evitar a radiação espalhada em órgãos de risco. Sendo assim, evita a radiação na medula, reto e bexiga.

A terapia da próstata usando feixes de prótons terapia, trás inúmeros benefícios aos pacientes. Alguns deles estão relacionados a não invasão dos tecidos, sendo mais precisa que outros tratamentos, proporcionam uma maior dose no tumor e possui menor risco para os efeitos colaterais causados pelo uso das radiações ionizantes.

Figura 2 - Gráfico do comparativo entre as duas formas de terapia.

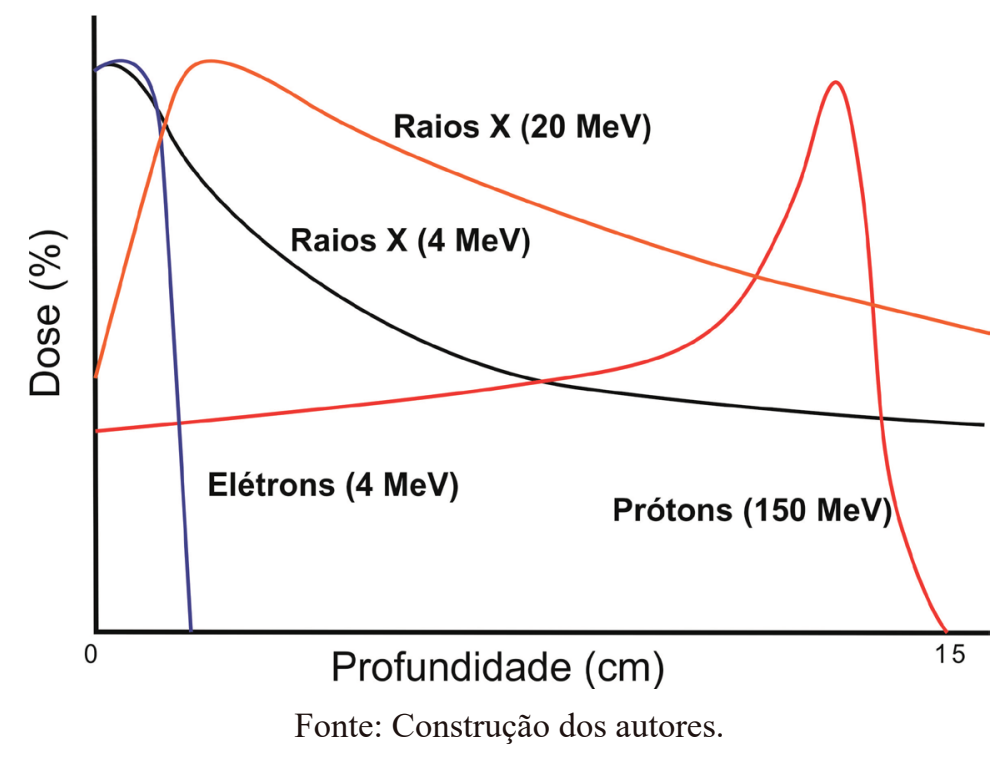

\section{CONCLUSÃO}

Houve um grande salto tecnológico na radioterapia, começando com os equipamentos de cobalto-60 com planejamento em 2D, avançando para aceleradores lineares, planejamento 3D-CRT, IMRT até a protonterapia, ainda não muito difundida por causa de seu valor elevado.

A protonterapia possui diversas vantagens em relação à terapia com fótons no tratamento do câncer de próstata, pois é mais precisa, não requer tempo de recuperação, apresenta riscos mínimos de impotência e menores efeitos colaterais em geral, possibilita doses mais altas para o tratamento 
sem danificar tecidos saudáveis, oferecendo melhor qualidade de vida para o paciente, além de se equiparar quanto à dor e desconforto do paciente (MAZAL, 2010).

Levando em consideração a revisão sistemática realizada no trabalho, pode-se concluir que a técnica da radioterapia de intensidade modulada para o câncer de próstata, é mais vantajosa em relação ao 3D-CRT, já que a sua modulação da dose protege de forma mais eficiente os órgãos de risco, tais quais bexiga, reto e pelve.

Também é importante salientar o uso das nanopartículas de ouro radioativas como novas formas de tratamento em braquiterapia para câncer de próstata. Estudos com códigos de Monte Carlo demonstraram que essa nova técnica permite a entrega de doses mais altas e homogêneas na região tumoral, e menor dose nos tecidos adjacentes vizinhos, principalmente reto e uretra. Indicando assim, uma modalidade promissora para tratar o câncer de próstata.

\section{REFERÊNCIAS}

ABBASI, A.; SADIKOGLU, F.; HASSANZADEH, M. Effect of Au-197 nanoparticles along with Sm-153 radiopharmaceutical in prostate cancer from simulation method. J Cancer Res Ther, v. 15, n. 8, p. $42-46,2019$.

AL-YASIRI, A.Y.; WHITE, N.E.; KATTI, K.V.; LOYALKA, S.K. Estimation of tumor and local tissue dose in gold nanoparticles radiotherapy for prostate cancer. Rep Pract Oncol Radiother, v. 24, n. 3, p. 288-293, 2019.

COLLINS, A.T. et al. Prospective identification of tumorigenic prostate cancer stem cells. Cancer Research, v. 65, n. 23, p. 10946-10951, 2005.

CONNELL, P. P.; HELLMAN, S. Advances in Radiotherapy and Implications for the Next Century: A Historical Perspective. Cancer Research, v. 69, n. 2, p. 383-392, 2009.

COSTA, R.J. et al. Biomarcadores em câncer de pulmão, próstata e mama. Disciplinarum Scientia, Naturais e Tecnológicas, v. 16, n. 2, p. 169-176, 2015.

DEARNALEY, D. et al. Articles Conventional versus hypofractionated high-dose intensity-modulated radiotherapy for prostate cancer: 5-year outcomes of the randomised, non-inferiority, phase $3 \mathrm{CHHiP}$ trial. The Lancet Oncology, v. 17, n. 8, p. 1047-1060, 2016.

EINSTEIN IMRT, Radioterapia com intensidade modulada IMRT. Einstein, 2019. Disponível em: 
https://bit.ly/2xAtEqj. Acesso em: 09 jul. de 2019.

FARIA, S.L. et al. Quimioterapia concomitante à radioterapia no tratamento adjuvante do câncer da mama localizado. Revista Brasileira de Cancerologia, v. 47, n. 2, p. 153-158, 2001.

KHAN, F.M.; GIBBONS, J.P. Khan's The Physics of Radiation Therapy. 5. ed. LWW, 2014.

KRENGLI, M. et al. Three-dimensional surface and ultrasound imaging for daily IGRT of prostate cancer. Radiat Oncol. v. 11, n. 1, p. 159-166, 2016.

MAZAL, A. et al. La protonthérapie: bases, indications et nouvelles technologies. Bulletin du Cancer. v. 97, n. 7, p. 831-846, 2010.

MINISTÉRIO DA SAÚDE. Câncer de próstata: causas, sintomas, tratamentos, diagnóstico e prevenção. Ministério da Saúde, 2018. Disponível em: https://bit.ly/2XFy1LC. Acesso em: 12 jun. de 2019.

MOUNTRIS, A.K.; VISVIKIS, D.; BERT, J. DVH-Based Inverse Planning Using Monte Carlo Dosimetry for LDR Prostate Brachytherapy. Int J Radiat Oncol Biol Phys, v. 103, n. 2, p. 503-510, 2019.

NASR, A.M.; ELAWADY, R.A. Dosimetric comparison of 3D conformal conventional radiotherapy versus intensity-modulated radiation therapy both in conventional and high dose radiation for prostate carcinoma. Res. Oncology, v. 12, n. 1, p. 10-14, 2016.

OKUNO, E.; YOSHIMURA, E. Física das Radiações. 1. ed. São Paulo: Oficina de Textos, 2010.

OLIVEIRA, H. F. et al. Radioterapia de intensidade modulada (IMRT) para pacientes do SUS: análise de 508 tratamentos em dois anos de instalação da técnica. Radiologia Brasileira, v. 47, n. 6, p. 355-360, 2014.

IMRT, Radioterapia de Intensidade Modulada. Instituto Oncoguia, 2014. Disponível em: https:// bit.ly/3euiBj5/. Acesso em: 24 jun. 2019.

RCT, Radioterapia Conformacional Tridimensional. Instituto Oncoguia, 2014. Dispoível em: https://bit.ly/2VMikzN. Acesso em: 24 jun. 2019. 
Research. v. 11, n. 2, p. 59-67, 2019.

TAUHATA, L. et al. Radioproteção e Dosimetria: Fundamentos. 10 ed. Rio de Janeiro: IRB/CBPF, 2014. 